\title{
CORRECTION TO ABSOLUTE PROPER MOTION USING THE IAS-GALAXY MODEL
}

\author{
KAVAN U. RATNATUNGA \\ NASA Goddard Space Flight Center \\ Greenbelt, Maryland 20771 \\ U. S. A.
}

\begin{abstract}
The IAS-Galaxy model (Ratnatunga, Bahcall and Casertano 1989) is a software interface between theoretical models of the Galaxy and observed kinematic distributions. It has been developed for analysis of many kinematic catalogs to study global galactic structure. In addition, the IASG model can be used to estimate corrections needed to derive absolute parallax and absolute proper motion by evaluating, on a starby-star basis, the expected mean motion of the reference stars.

A theoretical Galaxy model is defined on an inertial coordinate frame.* Proper motions are measured in a reference frame defined by a fundamental catalog. The observed distribution of proper motions in star catalogs can be directly compared with the expected distributions evaluated using IASG to check the accuracy of the adopted reference frame in realizing the inertial coordinate frame in the sky.
\end{abstract}

\section{Introduction}

Standard astrometric reduction of an observation series over a number of years in a small region of sky gives a relative parallax and a relative proper motion, with associated errors of measurement, for each of the stars in the field of view.

The reference frame is defined using quasars/galactic nuclei and/or FK5 stars. It is rare to find a sufficient number of very distant reference objects in the same field of view as the program star whose parallax and/or proper motion is being measured, to estimate the absolute motion directly in the adopted reference frame.

Correction to derive absolute proper motion often needs to be based on the expected proper motions for the stars in the neighboring region of sky. With improvements in precision of the measurements reducing random errors, the accuracy of the correction to absolute is now important to avoid systematic errors.

\section{IASG Galaxy Model}

\subsection{GOALS}

The IAS-Galaxy model is an attempt to build our current picture of the Galaxy in a computer. The program is basically a "software telescope" that can project an assumed empirical or theoretical model of the distribution function to derive expected distributions

* The terminology used in this paper was edited to be consistent with that adopted by G. A. Wilkins in the concluding paper of this conference. 
of all directly observed quantities within any combination of selection limits. Currently, the model is, to a large extent, empirical with a gradual change to include more theoretical constraints.

The final goal is to converge on a model that fits many different sets of observations, cataloged within various selection criteria, and to use self consistently, the same model, to estimate the corrections needed to evaluate absolute proper motion and parallax.

\subsection{MODEL}

The model for the Galaxy is based on assumed density and kinematic distributions. The density model is derived from the observed colors and apparent magnitudes using a spectrophotometric calibration, which in turn is based on the parallax of the nearest stars. The calibration is extended to both fainter and brighter absolute magnitudes using colormagnitude diagrams of star clusters. The kinematic model is based on line-of-sight velocities that are distance independent, as well as independent of the constant of precession. The observed proper-motion distribution needs to be consistent with the density model as well as the kinematic model.

The reference system used to construct the reference frame for proper motions, includes an estimate for the constant of precession to remove the drift caused by the slow movement of the Earth's axis of rotation. This estimate must also be based on a kinematic Galaxy model. In most analyses the velocity distribution function was assumed isotropic. Eichhorn (1974) warns of the error that the observed anisotropy in velocity dispersions could introduce to the estimated precession constant.

Use of many different Galactic models for different aspects of the data processing, does not assure self consistency. The model assumed to evaluate the corrections needs to be consistent with the observed distributions. Simplifications are no longer needed to do the required computations. As our knowledge of the distribution function of our Galaxy is improved, we must feedback these improvements in estimating corrections to derive absolute parallax and absolute proper motion and to evaluate the constant of precession.

\subsection{EVALUATIONS}

Proper motions are measured in a reference frame, that is constructed using a reference system, which assumes a Galaxy model, defined on an inertial coordinate frame in the sky. IASG can be used to check the accuracy of the reference frame in realizing the inertial coordinate frame. For example, to check for any residual rotation of the adopted reference frame, relative to the inertial coordinate frame, about any axis, we first select stars that are located on an equatorial band in a spherical coordinate system with the pole along that axis. We then compare the observed distribution of the component of proper motion for stars perpendicular to this axis, with the expected distribution evaluated using IASG. A preliminary comparison using proper motions from the AGK3 (corrected using improved IAU 1976 constant of precession) is seen to fit with a zero point error less than one millisecond of arc per year, for the three principle axes of the Galactic coordinate system.

To estimate the correction required to derive absolute parallax and/or absolute proper motion, IASG evaluates independently for each star, the expected parallax and proper motion distributions. IASG uses all of the available data, such as the galactic coordinates and color-apparent magnitudes of the reference stars, and integrates over the error distribution of each observable and over the total expected range of any observable that is unavailable.

The expected distributions are evaluated by direct projection of the assumed distribution function to the observed plane. This is essential to estimate the optimum correction 
using maximum likelihood. Monte Carlo estimates can not be used within an iteration loop used to maximize the likelihood function.

\subsection{LIKELIHOOD}

Numerical testing shows that maximum likelihood give unbiased estimates for the correction needed to derive absolute parallax, even when the program star has a parallax comparable to those of the reference stars. Conventional estimates are significantly biased in such a case, which could arise when the limiting distances of parallax measurements are extended by observations from space.

For example, at high galactic latitude, consequent to the volume sampled and the disk density law, most disk stars are contributed at a distance of order $1 \mathrm{kpc}$ at all apparent magnitudes. Therefore when measuring stars with a parallax smaller than a few milliseconds of arc, the bias caused by not using maximum likelihood is numerically found to be of order a few tenths of a millisecond of arc. The bias also depends on the uncertainty in the absolute magnitude of the reference stars. The bias being larger when only apparent magnitudes or just a mean apparent magnitude is available for the reference stars.

Re-evaluation of the corrections used to derive absolute proper motion and absolute parallax requires all available information regarding the reference stars and should be published along with each proper motion or parallax measurement. The corrections can then be re-evaluated to be consistent with any revised distribution function or improved when more information is available on the reference stars.

\section{Summary}

The kinematic Galaxy model assumed to estimate the corrections used to derive absolute proper motion and absolute parallax, as well as the model used to estimate the constant of precession, needs to be self consistent with the observed distributions.

The IASG Galaxy model is useful to evaluate expected distributions of all directly observed quantities in any star catalog, as well as the expected probability distributions of proper motions and parallax for each reference star, within the available information.

When the proper motions and parallaxes of the reference stars are comparable to those of the program stars, and measurement errors are not dominant, a detailed statistical analysis is important. Maximum likelihood gives an optimum way to use all available information, including individual error distributions of the observables. Using the full expected distribution and not just a tabulated mean, this procedure gives unbiased estimates of the correction required to derive absolute proper motion and parallax.

\section{Reference}

Ratnatunga, K. U., Bahcall, J. N., and Casertano, S. 1989, Astrophys. J. 339, 106. Eichhorn, H. 1974, Astronomy of Star Positions (New York: Frederick Ungar), 92.

\section{Discussion}

MURRAY: Can you describe the main components of your assumed model of galactic kinematics; in particular, did you allow for a shear in rotation velocity with height above the plane?

RATNATUNGA: The Galaxy is modeled as a sum of discrete density components, each of which is assumed to be isothermal with a mean assymetric drift. The change in the rotation velocity with height from the plane will arise naturally from change in the mixing proportions of the density components with height. 